\title{
Comportamiento mecánico de los nuevos instrumentos de endodoncia
}

Mechanical behavior of the new endodontic instruments
Comportamento mecânico dos novos instrumentos endodônticos
Fecha de Recepción

21 de diciembre 2018
Aceptado para su publicación

20 de marzo 2019
Carlos Daniel Lugo De Langhe

Asignatura Endodoncia. Módulos Clínica Rehabilitadora I y II. Facultad de Odontología. UNNE.

Mariel Beatriz Galiana

Asignatura Endodoncia. Módulos Clínica Rehabilitadora I y II. Facultad de Odontología. UNNE.

\section{Natalia Belén Montiel}

Asignatura Endodoncia. Módulos Clínica Rehabilitadora I y II. Facultad de Odontología. UNNE.

\section{Graciela Mónica Gualdoni}

Asignatura Endodoncia. Módulos Clínica Rehabilitadora I y II. Facultad de Odontología. UNNE.

\section{Autor de Correspondencia:}

Carlos Daniel Lugo De Langhe

Facultad de Odontología. UNNE. Av. Libertad 5450. Corrientes (Capital).

República Argentina.

Email: cdlugo@odn.unne.edu.ar

\section{Resumen}

En los últimos años han aparecido en el mercado una variedad de nuevos instrumentos de níquel-titanio, con distintos diseños, cinemáticas, secciones transversales y aleaciones. Estos mejoran la terapéutica endodóntica, disminuyendo significativamente la posible fractura de los mismos. El objetivo de este trabajo es realizar una revisión actualizada de los nuevos sistemas mecanizados de endodoncia y compararlos en relación a su resistencia a la fractura. Se realizó una búsqueda bibliográfica extensa en distintas bases de datos, limitando esta búsqueda a los últimos 6 años. Es necesario considerar la gran cantidad de sistemas mecanizados que han surgido en los últimos años, ya que las mejoras logradas en los mismos aumentarían la resistencia a la fatiga cíclica y torsional, evitando innumerables accidentes.

Palabras Claves: Fracturas Espontáneas, Aleaciones, Torsión Mecánica, Endodoncia.

\section{Abstract}

In recent years, a variety of new nickel-titanium instruments have appeared on the market, with different designs, kinematics, cross sections and alloys. They improve the endodontic treatment thus reducing significantly possible fractures. The objective of this work is to perform an updated review of the new mechanized endodontic systems and to compare them considering their 
resistance to fracture. An extensive literature search was conducted in different databases, limiting this search to the last 6 years. It is necessary to consider the large number of mechanized systems that have appeared in recent years, since the improvements achieved in them would increase the resistance to cyclic and torsional fatigue, avoiding innumerable accidents.

Key Words: Fractures Spontaneous, Alloys, Torsion Mechanical, Endodontics.

\section{Resumo}

Nos últimos anos, uma variedade de novos instrumentos de níquel-titânio surgiram no mercado, com diferentes desenhos, cinemática, seções transversais e ligas, que melhoram o tratamento endodôntico, reduzindo significativamente a fratura. $\bigcirc$ objetivo deste trabalho é realizar uma revisão atualizada dos novos sistemas endodônticos mecanizados e compará-los em relação à sua resistência à fratura. Uma extensa pesquisa bibliográfica foi realizada em diferentes bases de dados, limitando essa busca aos últimos 6 anos. É necessário considerar o grande número de sistemas mecanizados que surgiram nos últimos anos, já vez que as melhorias alcançadas nos mesmos aumentariam a resistência à fadiga cíclica e de torção, evitando inúmeros acidentes.

Palavras chave: Fraturas Espontâneas, Ligas, Torção Mecânica, Endodontia.

\section{Introducción}

La preparación mecánica y la desinfección química del conducto radicular (CR) no pueden considerarse por separado y se la denomina preparación químico-mecánica o biomecánica, que consiste básicamente en la conformación del sistema endodóntico, junto con la eliminación de la mayor parte de su contenido, consistente en tejido pulpar, restos necróticos, biofilms bacterianos o incluso obturaciones incorrectas de tratamientos previos'.

La búsqueda de una mejor preparación biomecánica del complejo sistemas de conductos ha llevado a varios avances en endodoncia, uno de ellos es la introducción de los instrumentos rotativos de níquel-titanio $(\mathrm{NITI})^{2}$.

Se demostró que el uso de los instrumentos de NITI mejoró la calidad de conformación de los CR, pero a su vez estos poseen limitaciones $y$ pueden ocasionalmente fracturarse en el interior de los conductos, poniendo en peligro el éxito del tratamiento, esto puede ser causado básicamente por dos mecanismos: la fatiga cíclica y torsional ${ }^{3,4}$. La fatiga por torsión se produce cuando un instrumento se bloquea en el $C R$ mientras el vástago sigue girando, mientras que la fatiga cíclica se produce cuando el instrumento gira dentro del conducto curvo sobre un número excesivo de ciclos de deformación tensióncompresión en la región de máxima curvatura, lo que desintegra su estructura y consecuentemente conduce a la fractura ${ }^{5,6}$. De acuerdo a esto, se puede inferir que el comportamiento mecánico de un instrumento es la suma de su resistencia a la torsión y flexión ${ }^{3}$.

El comportamiento del NITI superelástico en la tensión uniaxial a temperatura ambiente se puede dividir en cuatro etapas, la primera corresponde a la deformación elástica de la austenita (la fase cristalina a temperatura ambiente del NITI), la segunda etapa a la transformación a martensítica inducida por estrés, la tercera a la deformación elástica de la martensita y la última a la deformación plástica de la martensita. Siendo que los instrumentos endodónticos deben recuperar su forma original después del uso, los instrumentos deben alcanzar solo hasta la tercera fase ${ }^{3}$.

Esta superelasticidad se produce por una transformación de fase reversible entre austenita y martensita. Las propiedades mecánicas y el comportamiento del NITI, pueden ser alterados por pequeños cambios en la composición, impurezas y tratamiento térmicos durante el proceso de fabricación, esta característica del NITI ha creado una revolución en la fabricación de instrumentos endodónticos ${ }^{7}$.

Para aumentar la resistencia a la fractura de los instrumentos NITI, los fabricantes han introducido el uso de movimiento alternativo $y$ los instrumentos hechos de nuevas aleaciones, como M-Wire y la fase R NITI, cuya resistencia a la fatiga cíclica es supuestamente superior a las limas convencionales 5 . 
REVISTA FACULTAD DE ODONTOLOGÍA

ISSN 1668-7280 / ISSN-E 2683-7986

26

El objetivo de este trabajo fue realizar una revisión actualizada de los nuevos sistemas mecanizados de endodoncia y compararlos en relación a su resistencia a la fractura.

\section{Metodología}

Entre febrero y agosto de 2018 se realizó una búsqueda bibliográfica en las bases de datos Medline (PubMed) y EBSCOhost. Se limitó la búsqueda sobre publicaciones académicas de texto completo, publicados entre los años 2012 2018. Se utilizaron para la búsqueda los términos: instrumentation, canals, files endodontics, files endodontics and fracture, cyclic fatigue, new alloys in endodontics. Los criterios de inclusión fueron: artículos que contenían información sobre las características de fabricación, comportamiento mecánico de los instrumentos, estudios in vivo e in vitro y artículos sobre sistemas mecanizados que se consigan en el mercado argentino. Los criterios de exclusión que se utilizaron fueron: artículos de fractura de instrumentos de endodoncia por accidentes (iatrogenia) o uso inadecuado de protocolos clínicos y sistemas mecanizados que no hayan sufrido mejoras en su diseño, así como instrumentos reconocidos a nivel mundial pero que no se encuentren disponibles en el mercado local.

\section{Sistema Mecanizados}

Muchos sistemas mecanizados que han aparecido en el mercado hace varios años se siguen ocupando actualmente. Algunos como se expresa en la Tabla I, han evolucionado con mejoras que aumentan sus propiedades mecánicas.

\section{ProFile Vortex y Vortex Blue}

El sistema ProFile Vortex (Dentsply, Tulsa Dental Specialties, Tulsa, OK, EE.UU.) se fabrica a partir de una aleación M-Wire NITI, se comercializa de tamaños que van de \#15 a \#50 con conicidades 0,04 y 0,06 . Los instrumentos rotatorios ProFile Vortex tienen una sección transversal triangular $y$ un ángulo helicoidal específico y una punta de seguridad no cortante ${ }^{8,9}$.
El sistema Vortex Blue (Dentsply Tulsa Dental Specialties), tienen características de diseño similares a las Vortex hechas de M-Wire NITI, pero a diferencia de estas, las Vortex Blue tienen un color azul, que es el resultado de un proceso de fabricación patentado que crea una capa superficial de óxido. Se ha reportado que estas limas tienen propiedades mejoradas que aumentan su resistencia a la fatiga y flexibilidad en comparación con las Vortex de M-Wire ${ }^{10}$.

Shen et al.", analizaron la incidencia y el modo de los defectos del instrumento ProFile Vortex y Vortex Blue luego del uso clínico en un programa de endodoncia de posgrado, llegando a la conclusión que el riesgo de fracturas de las limas ProFile Vortex y Vortex Blue es muy bajo cuando los instrumentos son descartados después de un máximo de 3 casos.

Plotino et al. ${ }^{12}$, evaluaron la diferencia en la resistencia a la fatiga cíclica entre Vortex Blue (Dentsply Tulsa Dental, Tulsa, OK) y ProFile Vortex (Dentsply Tulsa Dental), donde sus resultados demostraron que Vortex Blue tuvo un aumento significativo en la resistencia a la fatiga cíclica en comparación con los mismos tamaños de ProFile Vortex.

\section{ProTaper Universal, ProTaper Gold y ProTaper Next}

El sistema Protaper Universal (PTU) y Protaper Gold (PTG, Dentsply Maillefer, Baillagues, Suiza), poseen una sección transversal triangular convexa con conicidad progresiva variable, la diferencia entre estos instrumentos radica en que el PTG es fabricado por una tecnología patentada de metalúrgica avanzada a través del tratamiento térmico, que según el fabricante aporta mayor flexibilidad y resistencia a la fatiga cíclica ${ }^{13}$.

Los Instrumentos Protaper NEXT (Dentsply Maillefer, Ballaiges, Suiza) están hechos de MWire NITI, se caracterizan por una innovadora sección transversal rectangular única descentrada, con excepción de los $3 \mathrm{~mm}$ apicales del instrumento $X I$ donde la sección es cuadrada. Esta característica de eje asimétrico descentrado da un movimiento serpenteante mientras avanza en la luz del conducto, donde el fabricante afirma que la rotación de esta sección transversal genera un mayor espacio para la eliminación de 
desechos. Están disponibles en tamaño \#17, conicidad 0,04; tamaño \#25, conicidad 0,06; tamaño \#30, conicidad 0,07 ; tamaño \#40, conicidad 0,06 ; y el tamaño $\# 50$, conicidad 0,06 ; tienen una conicidad variable al igual que su ahusamiento que se produce a lo largo de su parte activa. Estos instrumentos están fabricados a partir de aleaciones $M$, que produce una microestructura que contiene porciones de martensita, con lo que se pretende mejorar la flexibilidad del instrumento y la resistencia a la fatiga cíclica a la vez que conserva la capacidad de corte $\mathrm{e}^{14,15}$.

Uygun et al. ${ }^{16}$, compararon la resistencia a la fatiga cíclica de ProTaper Gold (PTG, Dentsply Tulsa Dental Specialties, Tulsa, OK, EE.UU.), ProTaper Next (PTN, Dentsply Tulsa Dental Specialties) y ProTaper Universal (PTU, Dentsply Tulsa Dental Specialties) a diferentes niveles, y sus resultados demostraron que los instrumentos PTG fueron los más resistentes a 5 y $8 \mathrm{~mm}$ de la punta; sin embargo, a $8 \mathrm{~mm}$ no hubo diferencias entre los instrumentos PTG y PTN. Los instrumentos PTU presentaron la menor resistencia a los ciclos de fractura en todos los niveles.

Hieawy et al. ${ }^{17}$, en este estudio examinaron el comportamiento de flexibilidad y fatiga cíclica del sistema ProTaper Gold (PTG) y compararlo con su predecesor el ProTaper Universal (PTU) y a su vez evaluaron el comportamiento de transformación de fase de estos instrumentos usando el análisis de calorimetría diferencial de barrido (DSC). Sus resultados demostraron que los instrumentos PTG fueron significativamente más flexibles y resistentes a la fatiga que los PTU. PTG mostró un comportamiento diferente de transformación de fase que PTU, lo que puede atribuirse al historial especial de tratamiento térmico de los instrumentos PTG. PTG puede ser más adecuado para la preparación de conductos con una curvatura más abrupta.

\section{Hyflex CM y Hyflex EDM}

El sistema Hyflex CM (Coltene-Whaledent) posee una sección triangular simétrica y son producidos por una metodología innovadora que utiliza un proceso único para controlar la memoria del material (un complejo tratamiento de calentamiento y enfriamiento), que utiliza una aleación equiatómica compuesta por $52 \%$ en peso de níquel, mientras que la mayoría de los sistemas comerciales están compuestos por $54,5-57 \%$ en peso de níquel, todo esto hace que el fabricante asegure que el sistema proporciona una flexibilidad superior y permitiría un mejor mantenimiento de la curvatura del $C R^{18,20}$.

El sistema HyFlex EDM (Coltene I Whaledent, Altstätten, Suiza), que se somete a tratamiento con memoria controlada (CM), ha demostrado que aumenta la flexibilidad y la resistencia a la fatiga cíclica, pero además son actualmente los únicos instrumentos producidos por el mecanizado de la descarga eléctrica, electrocución (EDM). Este instrumento se caracteriza por tener una sección transversal variable, es rectangular en la parte apical y se transforma en 2 formas trapezoidales diferentes en las porciones media y coronal ${ }^{2 !}$.

Kaval et al., ${ }^{22}$ realizaron un estudio donde evaluaron la fatiga cíclica y la resistencia a la torsión de los instrumentos Hyflex EDM, ProTaper Gold (PTG) y ProTaper Universal (PTU), llegando a la conclusión que las limas de Hyflex EDM demostraron una resistencia significativamente mayor a la fatiga cíclica. Aunque PTG y PTU tienen un diseño transversal similar, los instrumentos de PTG presentaron mayor resistencia a la fatiga cíclica y a la torsión que los instrumentos PTU. Las propiedades mejoradas de aleación de PTG podrían considerarse como la razón principal de esas diferencias.

Pedullà et al.,. ${ }^{23}$ evaluaron la resistencia a la fatiga torsional y cíclica del Hyflex EDM (Coltene / Whaledent AG, Altstatten, Suiza) comparándolo con Reciproc R25 (VDW, Múnich, Alemania) y WaveOne Primary (Dentsply Maillefer, Ballaigues, Suiza), concluyendo que los instrumentos Hyflex EDM tienen mayor resistencia a la fatiga cíclica y ángulo de rotación a la fractura, que Reciproc R25 y WaveOne Limas.

\section{WaveOne (Dentsply/Maillefer) y Wa- veOne Gold (WOG, Dentsply Maillefer)}

Los instrumentos WaveOne (Dentsply/Maillefer), con dos secciones transversales diferentes, de $\mathrm{D} 0$ a D8, tienen una forma triangulo-convexa modificada a modo de hélice, en tanto de D8 a DI6 es netamente triangulo-convexa. Posee 
REVISTA FACULTAD DE ODONTOLOGIA

ISSN 1668-7280 / ISSN-E 2683-7986

28

tres calibres, de \#21-0,06 (amarillo), \#25-0,08 (rojo), \#40-0,08 (negro). Vienen en longitudes de 21,25 y $31 \mathrm{~mm}$. Los fabricantes recomiendan un glide path inicial de al menos un instrumento numero \#10 antes de utilizar el instrumento ${ }^{24}$.

El sistema WaveOne Gold (WOG, Dentsply Maillefer) utiliza el mismo movimiento alternativo que las WaveOne originales, pero tienen una sección transversal modificada, tamaño y geometría distintos. Las WOG son ahora un paralelogramo con I o 2 filos cortantes dependiendo de la ubicación a lo largo de la lima, estos bordes están diseñados para minimizar el efecto de atornillado en las paredes del conducto, reduciendo en gran medida el torque mejorando la eficiencia del corte y permitiendo una mejor eliminación de los desechos, además de contar con un diseño descentrado como las ProTaper Next (Dentsply Maillefer). Las limas WOG se fabrican con metalúrgica avanzada y con una tecnología de tratamiento térmico, su color oro único viene de un procedimiento de ciclo térmico (calentado y enfriado lentamente muchas veces). Según el fabricante la resistencia a la fatiga cíclica de WOG Primary se ha mejorado en un $50 \%$ respecto de a WaveOne Primary y ahora es dos veces más resistente que la mayoría de los sistemas rotativos estándar, reduciendo aún más el riesgo de separación de las limas ${ }^{25}$.

Özyürek, ${ }^{26}$ comparó la resistencia a la fatiga cíclica de los instrumentos Reciproc R25, WaveOne Primary y WaveOne Gold Primary, donde el estudio demostró que la resistencia a la fatiga cíclica del sistema WaveOne Gold Primary fue mejor que la de los instrumentos WaveOne Primary y Reciproc R25.

Adıgüzel et al., ${ }^{27}$ compararon la resistencia a la fatiga cíclica de WaveOne (Dentsply Maillefer, Ballaigues, Suiza) y WaveOne Gold (Dentsply Maillefer) en sus tres tamaños (small, primary y large), llegando a la conclusión que las limas WoveOne Gold presentaron un mejor comportamiento en los tres tamaños analizados a comparación de la WaveOne.

\section{Reciproc y Reciproc Blue}

El sistema Reciproc Blue es un nuevo sistema de la VDW, que presenta las mismas características que el Reciproc original, tanto en sus longitudes, conicidades, números, pero se diferencia en el tipo de aleación NITI. Este presenta una nueva aleación NITI Blue Wire lograda a través de un avanzado tratamiento térmico, que otorga un color azul característico a estos instrumentos y que según la VDW mejora las características mecánicas de la lima ${ }^{28}$.

Gündoğar et al., ${ }^{29}$ compararon las resistencias a fatiga cíclica de Reciproc Blue (VDW, Múnich, Alemania), HyFlex EDM (Coltene / Whaledent, Altstätten, Suiza), WaveOne Gold (Dentsply Maillefer, Ballaigues, Suiza) y OneShape (Micro Mega, Besancon, Francia), y sus resultados demostraron que los instrumentos HEDM resistieron la fatiga cíclica estática significativamente más que los instrumentos OS, RPC Blue y WOG. El nuevo instrumento rotativo $\mathrm{NiTi}$ Reciproc Blue mostró una mejor resistencia a la fatiga cíclica que los instrumentos WOG y OS.

\section{XP-Endo Shaper}

El XP-Endo Shaper (FKG) posee una aleación única MaxWire que según los fabricantes le confiere al instrumento una flexibilidad excepcional y una resistencia extrema a la fatiga cíclica. Es un sistema de un solo instrumento, su sección transversal es triangular, $y$ posee la capacidad de comenzar a conformar con un diámetro ISO de \#15 y alcanzar el diámetro \#30, y a su vez aumenta su ahusamiento con conicidad de 0,0 I a por lo menos 0,04 , logrando una preparación final de \#30/04

Adiguzel et al., ${ }^{31}$ comparó la resistencia a la fatiga cíclica de los instrumentos rotatorios XPendo, HyFlex CM, FlexMaster y Race a la temperatura corporal $\left(37 \pm 1^{\circ} \mathrm{C}\right.$ ), los instrumentos se evaluaron en conductos artificiales con un radio de curvatura de $3 \mathrm{~mm}$ y un ángulo de curvatura de $60^{\circ}$ respecto al centro del canal de I,5 mm de ancho. Se giró cada instrumento hasta que se produjo la fractura y se registró el número de ciclos hasta el fallo, llegando a la conclusión que los instrumentos XP-Endo Shaper fueron más resistentes a la fatiga cíclica que los demás sistemas. 
Tabla I. Sistemas Mecanizados

\begin{tabular}{|c|c|c|c|c|c|}
\hline Instrumentos & Fabricante & $\begin{array}{c}\text { Sección } \\
\text { Transversal }\end{array}$ & Aleación & Movimiento & Fabricación \\
\hline Profile Vortex ${ }^{8,9}$ & Dentsply & Triangular & M-Wire & Continuo & Torneado \\
\hline Vortex Blue ${ }^{10}$ & Dentsply & Triangular & Blue-Wire & Continuo & Torneado \\
\hline $\begin{array}{l}\text { ProTaper } \\
\text { Universal }^{13}\end{array}$ & Dentsply & $\begin{array}{l}\text { Triangular } \\
\text { Convexa }\end{array}$ & NITI SE & Continuo & Torneado \\
\hline $\begin{array}{l}\text { ProTaper } \\
\text { Gold }{ }^{13}\end{array}$ & Dentsply & $\begin{array}{l}\text { Triangular } \\
\text { Convexa }\end{array}$ & Gold Wire & Continuo & Torneado \\
\hline $\begin{array}{l}\text { Protaper } \\
\text { Next }^{14,15}\end{array}$ & Dentsply & Rectangular & M-Wire & Continuo & Torneado \\
\hline Hyflex $C M^{18-20}$ & Coltene & Triangular & CM-Wire & Continuo & Torneado \\
\hline Hyflex EDM ${ }^{21}$ & Coltene & $\begin{array}{l}\text { Rectangular en } \\
\text { tercio apical } \\
\text { Trapezoidal } \\
\text { tercio medio } \\
\text { Triangular en } \\
\text { tercio cervical }\end{array}$ & CM-Wire & Continuo & $\begin{array}{c}\text { Mecanizado } \\
\text { por descarga } \\
\text { Eléctrica }\end{array}$ \\
\hline Wave One ${ }^{24}$ & Dentsply & $\begin{array}{l}\text { Triangulo } \\
\text { Convexa }\end{array}$ & M-Wire & Reciprocante & Torneado \\
\hline $\begin{array}{c}\text { Wave One } \\
\text { Gold }^{25}\end{array}$ & Dentsply & Paralelogramo & Gold-Wire & Reciprocante & Torneado \\
\hline Reciproc $^{28}$ & VDW & En "S" & M-Wire & Reciprocante & Torneado \\
\hline Reciproc Blue ${ }^{28}$ & VDW & En "S" & Blue-Wire & Reciprocante & Torneado \\
\hline $\begin{array}{l}\text { XP- Endo } \\
\text { Shaper }\end{array}$ & FKG & Triangular & Max-Wire & Continuo & Torneado \\
\hline
\end{tabular}

\section{Conclusiones}

En los últimos años, han salido al mercado instrumentos con innumerables modificaciones en sus diseños, según la evidencia científica analizada es evidente que el cambio que más aumentaría la resistencia a la fractura, es la modificación de la aleación tradicional por las nuevas tratadas térmicamente, es por esto que se recomienda el uso de estos instrumentos con aleaciones mejoradas para el tratamiento de conductos con curva severa. 
REVISTA FACULTAD DE ODONTOLOGÍA ISSN 1668-7280 / ISSN-E 2683-7986

30
DIVULGACIÓN

Lugo De Langhe - Galiana - Montiel - Gualdoni

\section{Referencias Bibliográfícas}

I. Stefanescu T, Antoniac IV, Popovici RA, Galuscan A, Tirca T. Ni-ti rotary instrument fracture analysis after clinical use. structure changes in used instruments. Environ Eng Manag J. 2016; I5 (5): 981-988.

2. Farmakis ETR, Sotiropoulos GG, Pantazis $\mathrm{N}$, Kozyrakis $\mathrm{K}$. The permanent deformation of the self-adjusting files when used in canals of extracted teeth. Int Endod J. 2013; 46 (9): 863-869.

3. Leroy AMF, de Azevedo Bahia MG, Ehrlacher A, Lopes Buono VT. An analytical mechanical model to describe the response of $\mathrm{NiTi}$ rotary endodontic files in a curved root canal. Mater Sci Eng C Mater Biol Appl. 2012; 32 (6): I594- 1600.

4. King JB, Roberts HW, Bergeron BE, Mayerchak MJ. The effect of autoclaving on torsional moment of two nickel-titanium endodontic files. Int Endod J. 2012; 45 (2): $|56-| 6 \mid$.

5. Ferreira F, Adeodato C, Barbosa I, Aboud L, Scelza P, Zaccaro Scelza M. Movement kinematics and cyclic fatigue of NiTi rotary instruments: a systematic review. Int Endod J. 2017; 50 (2): |43-| 52.

6. Montalvão $D$, Shengwen $Q$, Freitas $M$. A study on the influence of $\mathrm{Ni}-\mathrm{Ti} \mathrm{M}$-Wire in the flexural fatigue life of endodontic rotary files by using Finite Element Analysis. Mater Sci Eng C Mater Biol Appl. 2014; 40: I72-179.

7. Shen Y, Zhou HM, Zheng YF, Peng B, Haapasalo $M$. Current challenges and concepts of the thermomechanical treatment of nickel-titanium instruments. J Endod. 201 3; 39 (2): I63-I72.

8. Shen Y, Coil JM, Zhou HM, Tam E, Zheng YF, Haapasalo M. ProFile Vortex instruments after clinical use: a metallurgical properties study. Journal of endodontics. 2012; 38 (12): 1613-1617.
9. González Sánchez JA, Duran $\square$ Sindreu F, de Noé S, Mercadé M, Roig M. Centring ability and apical transportation after overinstrumentation with ProTaper Universal and ProFile Vortex instruments. International endodontic journal. 2012; 45 (6): 542-55I.

10. Duke F, Shen Y, Zhou H, Ruse ND, Wang ZJ, Hieawy A, et al. Cyclic Fatigue of ProFile Vortex and Vortex Blue Nickel-Titanium Files in Single and Double Curvatures. J Endod. 20I5; 4 I (10): I686-I690.

II. Shen Y, Zhou H, Coil JM, Aljazaeri B, Buttar R, Wang Z, et al. ProFile Vortex and Vortex Blue nickel-titanium rotary instruments after clinical use. J Endod. 2015; 41 (6), 937-942.

12. Plotino G, Grande NM, Cotti E, TestareIli L, Gambarini G. (20|4). Blue treatment enhances cyclic fatigue resistance of vortex nickel-titanium rotary files. J Endod. 2014; 40 (9): |45|-|453.

13. Plotino G, Grande NM, Mercadé M, Testarelli L, Gambarini G. (2016). Influence of Temperature on Cyclic Fatigue Resistance of ProTaper Gold and ProTaper Universal Rotary Files. J Endod. 2017; 43 (2): 200202.

14. Burklein S, Mathey D, Schafer E. Shaping ability of ProTaper NEXT, ProTaper Universal and iRace files in simulated S-shaped canals. Int Endod J. 201 5; 48 (8): 774-78I.

15. Zanesco C, Só MVR, Schmidt S, Camargo Fontanella VR, Grazziotin-Soares R, Barletta FB. Apical Transportation, Centering Ratio, and Volume Increase after Manual, Rotary, and Reciprocating Instrumentation in Curved Root Canals: Analysis by Micro-computed Tomographic and Digital Subtraction Radiography. J Endod. 20 17; 43 (3): 486-490.

16. Uygun AD, Kol E, Topcu MK, Seckin F, Ersoy I, Tanriver M. Variations in cyclic fatigue resistance among ProTaper Gold, ProTa- 
per Next and ProTaper Universal instruments at different levels. Int Endod J. 2016; 49 (5): 494-499.

17. Hieawy A, Haapasalo M, Zhou H, Wang ZJ, Shen Y. Phase transformation behavior and resistance to bending and cyclic fatigue of ProTaper Gold and ProTaper Universal instruments. J Endod. 20I5; 4I (7): II341138.

18. Koçak MM, Çiçek E, Koçak S, Sa $\square$ lam BC, Furuncuo $\square$ lu F. Comparison of ProTaper Next and HyFlex instruments on apical debris extrusion in curved canals Int Endod J. 2016; 49 (10): 996-1000.

19. Saber SE, Nagy MM, Schäfer E. Comparative evaluation of the shaping ability of ProTaper Next, iRaCe and Hyflex CM rotary $\mathrm{NiTi}$ files in severely curved root canals. Int Endod J.20| 5; 48 (2): |3|-| 36.

20. Alfoqom Alazemi M, Bryant ST, Dummer PMH. Deformation of HyFlex CM instruments and their shape recovery following heat sterilization. Int Endod J. 20I5; 48 (6): 593-60I.

21. Venino PM, Citterio CL, Pellegatta A, Ciccarelli M, Maddalone M. A Micro computed Tomography Evaluation of the Shaping Ability of Two Nickel-titanium Instruments, HyFlex EDM and ProTaper Next. J Endod. 2017; 43 (4): 628-632.

22. Kaval ME, Capar ID, Ertas H. Evaluation of the Cyclic Fatigue and Torsional Resistance of Novel Nickel-Titanium Rotary Files with Various Alloy Properties. J Endod. 2016; 42 (I2): I840-I843.

23. Pedullà E, Savio FL, Boninelli S, Plotino G, Grande NM, La Rosa G, et al. Torsional and cyclic fatigue resistance of a new nickel-titanium instrument manufactured by electrical discharge machining. J Endod. 2016; 42 (I): I56-I59.

24. Bürklein S, Hinschitza K, Dammaschke T, Schäfer E. Shaping ability and cleaning effec- tiveness of two single-file systems in severely curved root canals of extracted teeth: Reciproc and WaveOne versus Mtwo and ProTaper. International Endodontic Journal. 20 I 2; 45 (5): 449-46।.

25 . Introducing the gold standard. British Dental Journal [Internet]. 2015 Apr 24 [cited 2019 Mar 8]; 218 (8): 495. Available from: http://search.ebscohost.com/login.aspx?dir ect $=$ true $\& d b=e d b \& A N=102260665$ \&lang $=$ es\&site=eds-live.

26. Özyürek T. Cyclic fatigue resistance of Reciproc, WaveOne, and WaveOne Gold nickel-titanium instruments. J Endod. 2016; 42 (10): I536-I539.

27. Adigüzel M, Capar ID. Comparison of Cyclic Fatigue Resistance of WaveOne and WaveOne Gold Small, Primary, and Large Instruments. J Endod. 2017; 43 (4): 623627.

28. De-Deus G, Silva EJ, Vieira VT, Belladonna FG, Elias CN, Plotino G, et al. Blue Thermomechanical Treatment Optimizes Fatigue Resistance and Flexibility of the Reciproc Files. J Endod. 201 7; 43 (3): 462-466.

29. Gündo $\square$ ar M, \& Özyürek T. Cyclic Fatigue Resistance of OneShape, HyFlex EDM, WaveOne Gold, and Reciproc Blue Nickeltitanium Instruments. J Endod. 20। 7; 43 (7): II92-1196.

30. Bayram HM, Bayram E, Ocak M, Uygun AD, Celik HH. Effect of ProTaper Gold, Self-Adjusting File, and XP-endo Shaper Instruments on Dentinal Microcrack Formation: A Micro-computed Tomographic Study. J Endod. 2017; 43 (7): II66-I I69.

3I. Adiguzel M, Isken I, \& Pamukcu II. Comparison of cyclic fatigue resistance of XPendo Shaper, HyFlex CM, FlexMaster and Race instruments. J Dent Res Dent Clin Dent Prospects. 20I8; 12 (3): 208-2 I 2. 